\title{
A Multiagent-Based Model for Epidemic Disease Monitoring in DR Congo
}

\author{
Jean-Claude Tshilenge Mfumu ${ }^{1(\bowtie)}$, Annabelle $\operatorname{Mercier}^{2(\bowtie)}$, \\ Michel Occello ${ }^{2(\bowtie)}$, and Christine Verdier ${ }^{1(凶)}$ \\ ${ }^{1}$ Univ. Grenoble Alpes, CNRS, Grenoble INP, LIG, 38000 Grenoble, France \\ \{jean-claude. tshilenge-mfumu, \\ christine.verdier\}@univ-grenoble-alpes.fr \\ ${ }^{2}$ Univ. Grenoble Alpes, Grenoble INP, LCIS, 26000 Valence, France \\ annabelle.mercier, \\ michel.occello\}@univ-grenoble-alpes.fr
}

\begin{abstract}
Any infectious diseases have been reported in sub-Saharan countries over the past decade due to the inefficiency of health structures to anticipate outbreaks. In a poorly-infrastructure country such as the Democratic Republic of Congo (DRC), with inadequate health staff and laboratories, it is difficult to respond rapidly to an epidemic, especially in rural areas. As the DRC's health system has three levels (peripheral, regional and national), from the production of health data at the peripheral level to the national level that makes the decision, meantime the disease can spread to many people. Lack of communication between health centres of the same health zone and Health zones of the same Health Provincial Division does not contribute to the regional response. This article, an extended version of [1], proposes a well elaborated solution track to deal with this problem by using an agent-centric approach to study by simulation how to improve the process. A new experiment is described by arranging twentyeight health zones of Kinshasa to show how their collaboration can provide unique health data source for all stakeholders and help reducing disease propagation. It concerns also 47 health centres, 1 medical laboratory, 1 Provincial Health Division and 4 Rapid Riposte Teams. The simulation data, provided by Provincial Health Division of Kinshasa, concerned cholera outbreak from January to December 2017. The interaction between these agents demonstrated that Health Zone Agent can automatically alert his neighbours whenever he encountered a confirmed case of an outbreak. This action can reduce disease propagation as population will be provided with prevention measures. These interactions between agents have provided models to propose to the current system in order to find out the best that can help reducing decision time.
\end{abstract}

\section{Introduction}

Access to health care is a major concern in developing countries. The Democratic Republic of Congo ranks among the poorest countries according to its $\mathrm{HDI}^{1}$ [2]. Despite its millions of hectares of arable land, this vast country of Central Africa is

\footnotetext{
${ }^{1}$ Human Development Index. 
experiencing serious difficulties in improving the living conditions of its population, particularly in the field of basic health care. Life expectancy at birth is 50 and 53 respectively for men and women [3].

The country is currently divided into city-province of Kinshasa and 25 other provinces. The provinces are subdivided into territories which are divided into sectors. To facilitate the supervision of health structures, DRC health system is divided into three levels [4]: central, intermediate and peripheral. The nearest level to population is the peripheral area composed of 518 health zones (HZ) that coordinate the actions of health facilities. A Health Zone is divided into Health Area (HA) containing one or more Health Centers (HC) and a General Referral Hospital (GRH). The Central (national) level defines the policies, strategies and resources of the sector. It enforces strategies and policies at the peripheral level through the intermediate level called the Provincial Health Division (PHD), which coordinates primary health care and technical support activities for health zones in a province. Provincial Health Minister (PHM) is the political authority of the province.

Each Health Zone has a Health Information Bureau (HIB) which retrieves aggregated data from all its supervised Health Area to send to national level for decision measures. HIB has a Health Zone Executive Team (HZET) that organizes weekly meetings to discuss about suspicious cases to report to hierarchy if needed. Health Zone Executive Team manages health facilities (HF) that includes Health Center and GRH. Figure 1 represents three levels of DRC Health System in which each Health Zone Executive Team supervises many Health Center and one GRH at peripheral level. Provincial Health Division at intermediate level provides technical assistance to Health Zone Executive Team. Direction of Disease Control (DDC) at central level gives national policies to 26 Provincial Health Divisions. Provincial Health Minister plays also of political authority's role.

As WHO member country, DRC benefits from the technical and financial support of the partners to respond to epidemics under the conditions stipulated in the International Health Regulations (IHR) [5]. All cases of these four diseases must be automatically notified to WHO: smallpox, poliomyelitis due to wild-type poliovirus, severe acute respiratory syndrome (SARS) and cases of human influenza caused by a new subtype. On the ever-changing list of diseases provided by IHR, each country is free to add other diseases, with epidemic potential or not, which constitute a public health problem.

Access to basic care is difficult for a large part of population. People visit the health facilities in case of extreme emergency. This is more evident in rural areas where the diminishing resources of farmers do not allow them to consult medical services regularly. Most of time health care is provided during free medical workers' campaigns.

The most usual ways to collect data about cases that must be sent to the hierarchy for decision-making are described below [6]:

- Pharmacies must report when same medicines are increasingly purchased by population or when they detect some recurrent treatments;

- Schools reporting unusual rates of pupil absences due to strange signs and symptoms; 


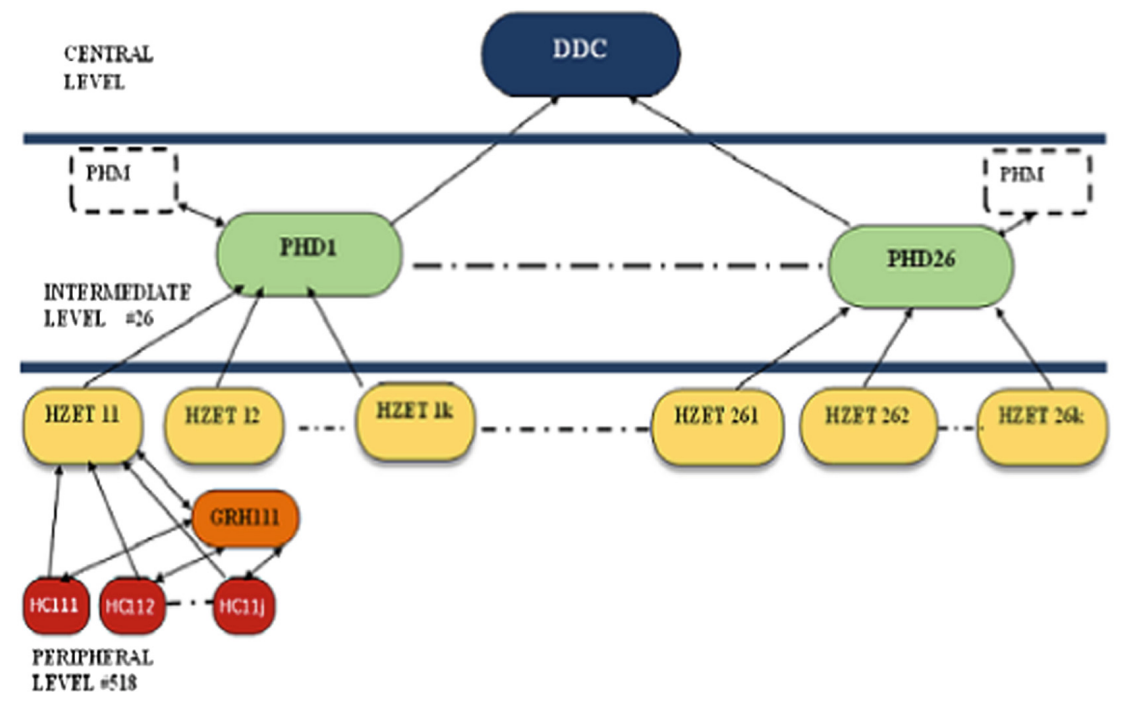

Fig. 1. Peripheral level contains health centre (HC) under the supervision of Health zone Executive Team (HZET). Provincial Health Division, the intermediate and Direction of Disease Control, the central or national level.

Figure 2 presents an exhaustive list of actors used in providing health data.

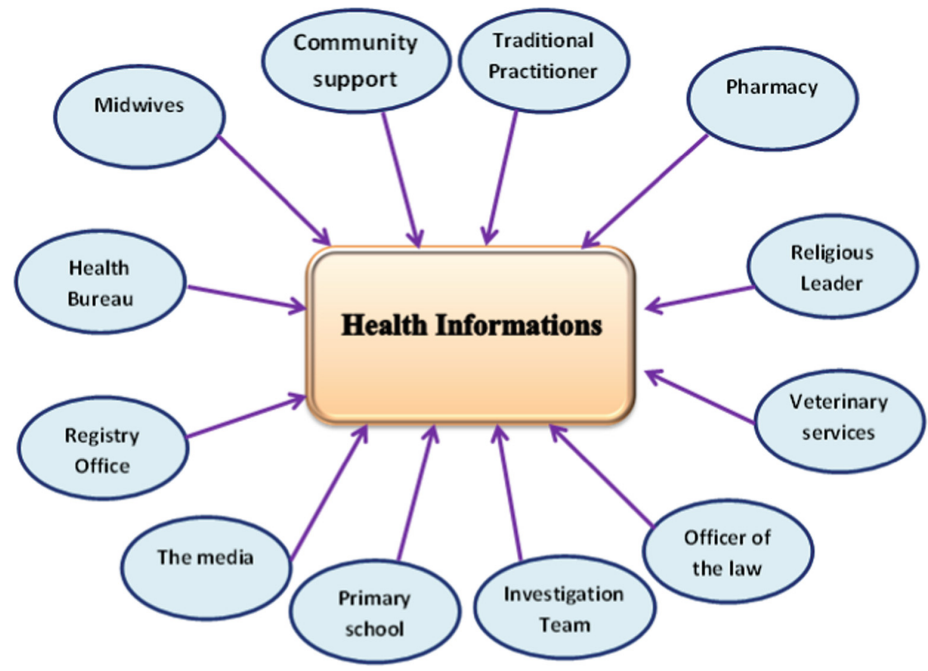

Fig. 2. Many structures are designated to produce Health data at each Health Zone [1].

Despite great efforts to improve disease surveillance and response, DRC faces big challenges in identifying, diagnosing and reporting infectious diseases properly due to 
the remoteness of communities, the inadequate transport and communication infrastructures, and the lack of qualified health staff and laboratory facilities to ensure accurate diagnosis.

The challenge, in this paper, is to find new solutions based on real population life and situation to improve health services organization and data sharing in order to detect infectious disease very quickly, organize the response and prevent the spread of disease.

\section{Issues}

In this paper, we present a part of this challenge. We propose a multi-agent system to simulate the interactions between actors working together to organize an optimal response to epidemic detection. When a new case of infectious disease is suspicious in Health Center, actors will collaborate to report it to Provincial Health Division through Health Zone Executive Team. The approach will be based on the current DRC heath system processes to extract relevant actors' tasks. The identification of these actors and their tasks will provide the opportunity to simulate a new system that distributes the entire competences of the old heath system to those actors in order to improve their collaboration and eventually shorten the making-decision time response. Work-sharing protocols will be proposed to simplify the complexity of the data sources.

This paper focuses precisely on improving the process of reporting health data from the peripheral level to the hierarchy for rapid decision-making and anticipate as much as possible the medical response using multi-agent systems (MAS). Hierarchical dependency between three levels forbids peripheral to directly transmit health data to National level for quick decision. As information must pass through intermediate level (Provincial Health Division), with defective means of communications, it drastically hampers the fight against a propagation of a disease. Since decision-making is pushed back to the central level, it can intervene belatedly at the risk of witnessing an alarming spread of an epidemic with high epidemic potential.

The next section shows the related work in healthcare and multiagent domains. Section 3 describes the healthcare system and problematic in DRC. The methodological approach and agent's models are explained in Sect. 4. The model is validated by a simulation presented in Sect. 5. Future research directions and conclusion are developed in Sect. 6.

\section{Related Work}

Information and Communication Technology is a powerful solution for health care in developing countries [7]. It made possible the improvement of remote patient follow-up [8], controlling the progression of malaria [9], improving the uptake of information from health systems [10]. Two main ways of research can be studied in this paper: the use of mobile phone as a relevant medium to rapidly transfer medical data and the multi-agent system that is powerful to simulate organizational skills to anticipate diseases spreading. 
Mobile phone coverage in Africa grew from 10\% in 1999, 65\% in 2008 to more than $70 \%$ in 2012 [11]. This technology is used to cover numeric fraction. To improve drug adherence and suppression of plasma HIV-1 RNA load in Kenyan, mobile phone communication between health-care workers and patients starting antiretroviral therapy was set up [12] Text-message reminders sent to health workers' mobile phones improved and maintained their adherence to treatment guidelines for outpatient pediatric malaria [13].

Phone traces are powerful tools to estimate population migration while investigating an outbreak. These techniques were used to demonstrate the feasibility of rapid estimates and to identify areas at potentially increased risk of outbreaks in Haiti. They produced reports on SIM card movements from a cholera outbreak area at its immediate onset and within $12 \mathrm{~h}$ of receiving data. Results suggest that estimates of population movements during disasters and outbreaks can be delivered rapidly and with potentially high validity in areas with high mobile phone.

A trial of mobile phone text messaging for diabetes management in an eight-month period to transmit data such as blood glucose levels and body weight to a server that automatically answered with a monthly calculated glycosylated hemoglobin result. The trial results suggest that sms may provide a simple, fast and efficient adjunct to the management of diabetes.

In developed countries SMS messages have been widely used to remind patients of scheduled appointments Car and all, 2008). Similarly, more complex mobile phone applications have shown significant improvement in the follow-up of malaria patients in Thailand. The same approaches have been tested in Africa as part of the SMS reminder package to improve patients' adherence to antimalarial treatment schedules in .six sub Saharan countries [9].

Even if text-messaging is the simplest and the most widely easy to use technology function in reporting periodic data from the health system peripheral to control managers, it however needs to be experienced in interventions targeting individual patients, for whom a high facility workload or illiteracy may present a barrier.

A Multi-agent System is a set of agents situated in a common environment, which interact and try to reach a set of goals. Through these interactions, a global behavior, more intelligent than the sum of the local intelligence of multiagent system components, can emerge. By 'agent' we mean a software entity evolving in an environment that it can perceive and within which it reacts. There are several kinds of agents. A reactive agent reacts to the stimuli of the environment. Cognitive agents have a more developed intelligence: they manage knowledge and make decisions according to their internal states and according to their perception of the environment. It is provided with autonomous behaviors and some objectives. Autonomy is the main concept in the agent issue: it is the ability of agents to control their actions and their internal states. The agents' autonomy implies no centralized control [14]. When a MAS integrates agents on the fly dynamically during the execution, the system is qualified as an opened one. Otherwise, it is a closed one. One of the advantages of MAS is to model systems where a global description is not possible at any given moment. Multi-agent conception is well suitable to model actors described in Fig. 1.

Simulation based on MAS approach has wide potential applications in healthcare. First case, MAS approaches are suitable to applications where a complete control is 
unreachable, the high number of entities or the complexity of entities' behavior make hard to represent the overall system. The second case is related to the monitoring of the epidemics. In a general way, the model of each agents going into action in the system is designed in microscopic level. The environment, the agents' interactions and the social organizations are defined at the macroscopic level. In MAS domain, there are numerous methods to approach the analysis and the design of the software application. The methods come from various domains such as object oriented design, knowledge engineering or reproduction of behavior or natural phenomena. The first methods were AAII [15] which uses an external view (roles services, organization) and internal view (agent design bases on Belief Desire Intention architecture). Cassiopée [15] is a bottom-up approach based on natural behavior and DESIRE [16] is based on knowledge engineering. The method Vowels [17] allows obtaining modularity at the level of the multi-agents models by decomposing the problem into four elementary facets. Other method like Gaia [18] extends the concepts used in classical object engineering and provides a microscopic and a macroscopic view of the software system. There are also complete approaches for developing multi-agents systems from the analysis to the deployment by using MaSE [19] or Prometheus [20]. Moreover some models al-low the designer to develop the agent like AGR [19] based on Agent/Group/Role or BDI [21] based on Belief/Desir/Intention.

The main concept of all those approaches is the agent and the communication between agents to lead to the main objective of the software.

The general classification is clinical, operational, managerial and educational simulation. Managerial and operational simulations are closely interrelated. Both are the core components for healthcare process management. Some challenges and trends of simulation models in healthcare in the past two decades have been developed The design of a web-based clinical decision support system that guides patients with Low Back Pain in making suitable choices on self-referral has been experienced in Netherlands.

MAS is used to describe an approach to the analysis and development of telemedicine systems [22], to manage communications in wireless sensor networks [23], the epidemiological decision support system [24], the care of seniors at home [25], decision-making for monitoring and prevention of epidemics [26], evaluation of disaster response system [27], medical sensor modules in conjunction with wireless communication technology supporting a wide range of services including mobile telemedicine, patient monitoring, emergency management and information sharing between patients and doctors or among the healthcare workers [28].

MAS can be considered as a suitable technology for the realization of applications providing healthcare and social services where the use of data and remote collaborations among users are often the most requirements [29]. Cooperation in Agent Technology can provide better healthcare than the traditional medical system [30]. Real programs built on the multiagent paradigm are still evolving towards a complete maturity. The variety and complexity of the e-health scenario make it one of the most interesting application fields, able to check the advantages of their use to condition their evolution [31].

MAS was used to monitor a generic medical contact center for chronic disease environment, detect important cases, and inform the healthcare and administrative 
personnel via alert messages, recommendations, and reports, prompting them to action [32]. Developed MAS applications in healthcare can provide a reasonable way to mitigate the cost due to increased demand for services [33].

An Agent-Based Model (ABM) with Geospatial and Medical Details was used to evaluate the efficiency of disaster responders to rescue victims in a mass casualty incident situation in South Korea [27].

ABM can cooperate to share tasks between sensors observing a phenomena [34], to manage diabetes treatment between Caregivers and Patients. The usability evaluation of a collaborative information system for dementia assessment built using a usercentered design approach was experienced in Norway [35]. But from several research papers we have reviewed we didn't find a paper addressing ABM in sharing tasks from multisource health information to organize a rapid response to a high epidemic potential disease.

\section{Health System in DRC}

\subsection{Administrative Structures}

The patient health care and the reporting of suspicious cases are managed at the peripheral level by Health facilities (HF): health centers plus the general referral hospitals. Health data collected by the Health Facilities are transmitted to Health Zone Executive Team for consolidation, analyzes and decision-making. Aggregated data from entire Health Zone are also transmitted to Provincial Health Division. Each week this intermediate level structure convenes meetings to analyze data from each Health Zone, decide on actions to take. Provincial Health Division produces consolidated data for its province.

Provincial Health Division must transmit the health data from its province to the central level for a second analysis and national consolidation. If suspicious cases reported by Health Zone require deeper investigation, laboratory tests or kits intervention, Provincial Health Division will ask for technical and financial supports from central level.

Disease Control Direction (DCD) is a central respondent at central level. It also organizes weekly meetings to analyze health data from all provinces. It often provides advice and recommendations to Provincial Health Division to monitor suspicious cases in accordance with the national policy of the sector. Whenever Provincial Health Division asks for a help, DCD can approach government authorities, special programs, partners and even the international community, to fill up needs. Difficulties encountered by Health Facilities to better report information and structures dependency are well expressed at next section.

\subsection{Structure Dependencies}

The first challenge in managing epidemics begins with the multi-sources data processing at the Health Zone level. National policy has provided list of groups of individuals who can retrieve information from suspicious cases. This information 
transmitting by phone calls or narrative is not exhaustive. Hence, the interest in diversifying the mode of communication by adding text and voice sms, tweets and phone calls on green lines can enhance data completeness.

A second difficulty in an accurate identification of suspicious cases is the insufficient number of qualified health staff [36]. Despite training courses organized by Health Zone Executive Team for community relays and staff of health facilities, there are gaps in the implementation of the information brought to their attention. For example, the Provincial Health Division can conduct a thorough investigation with qualified staff as soon as the number of suspicious cases reaches the threshold for the pathology. Lack of information on the list of the nearest laboratories delays response time to confirm cases and ensure accuracy of diagnosis.

Hierarchical dependences do not favor communication between structures of the same level such as health areas of a same Health Zone or of contiguous health areas but belonging to different Provincial Health Division. This lack of dialogue can lead to the non-detection of an epidemic for the simple reason that the number of suspicious cases to organize investigation is not reached in an Health Zone. However, by combining number of suspicious case found in contiguous health areas, we could detect the pathology at the intersection of the provinces.

\subsection{Collection and Response for Epidemic Surveillance}

Structures authorized to report information relating to suspicious cases to Health Zone Executive Team are Health Facilities. But, community relays can also report their observations that need to be considered by Health Facilities. Reports concern pathologies described at International Sanitary Regulations (smallpox, poliomyelitis due to wild polio virus, human influenza and Severe Acute Respiratory Syndrome (SARS)) and local list of diseases with epidemic Eradication measures or Elimination and other chronic diseases provided by authorities.

\subsection{Data Collection and Epidemics Response}

As soon as it appears, suspicious cases must be transmitted to Health Zone Executive Team by all data providers indicated on Fig. 2. When number of suspicious cases in Health Zone equals to the threshold of observed pathology, a rapid riposte team (RRT) has to investigate some Health Center and the population of the concerned Health Area to make sure the allegation was correct. The investigation of Rapid Ripost Team could result to laboratory tests of some samples. In case of riposte many hierarchical structures such as Provincial Health Division or national level would intervene to provide technical and financial supports.

The process used to organize riposte simulation is heavily based upon computer science, mathematics, probability theory and statistics: yet the process of simulation modeling and experimentation remains very much an intuitive art. Simulation is a very general and somewhat ill-defined subject. For the purpose of this paper, we will define simulation as «the process of designing a computerized model of a system and conducting experiments for the purpose of understanding the behavior of the system and/or of evaluating various strategies for the operation of the system. Thus we will 
understand the process of simulation to include both the construction of the model and the analytical use of the model for studying a problem shortly described in Fig. 3 . Health Zone Executive Team analyze the report of surveillance to determine if the number of suspicious case has reached the threshold to order an investigation. Rapid Ripost Team will research new cases at Health Area according to the clinical definition of case. It will find out new determinants of the outbreak to report to Provincial Health Division in other to realize the response.

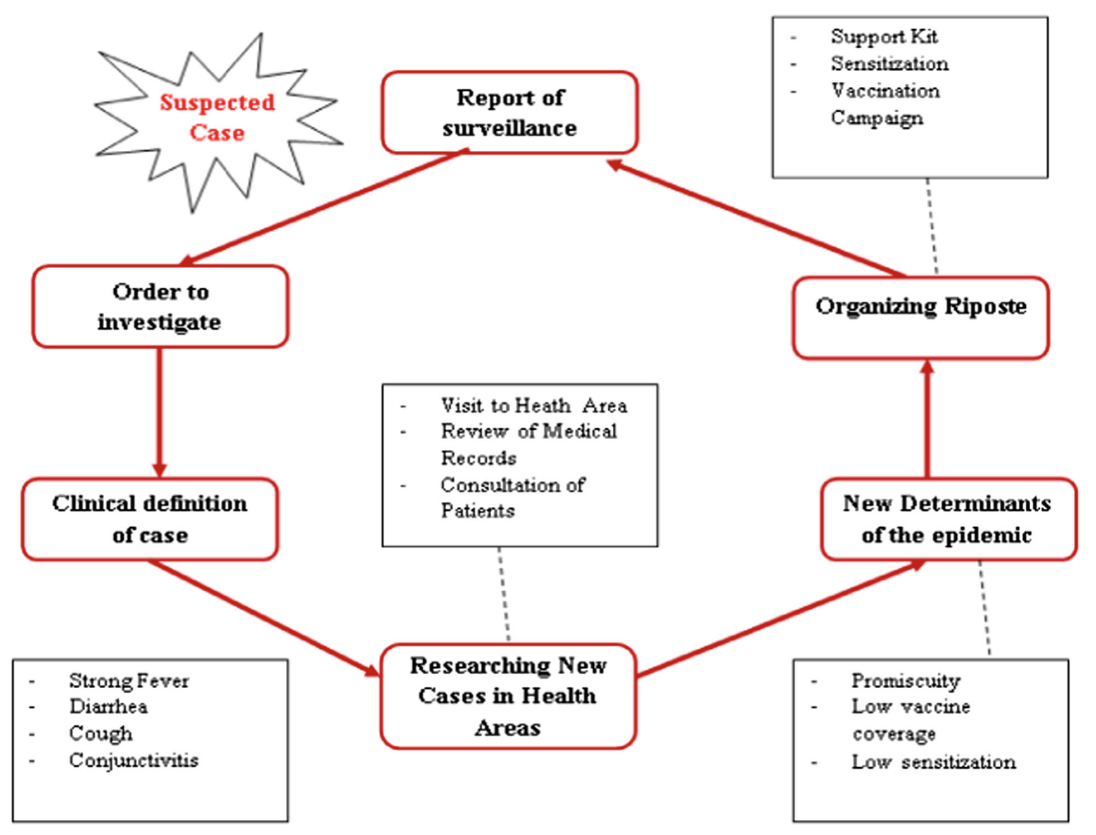

Fig. 3. Cycle of outbreak response [1].

A final evaluation of outbreak response presented as a report of the process can be shared with other Health Zone and Health Facilities.

This type of system is well suited to MAS using an AEIO representation. Real system is analyzed with four elements: Agent, Environment, Organizations and Interactions between agents. This model will be detailed in the next part.

\section{Individual-Centered Models}

The hierarchical organization of the healthcare system in DRC is a good candidate for a multiagent model because there are several kinds of agents with personal goals sharing the same global achievement. In the process described in Fig. 3, the agents use some knowledge and tasks to perform a main goal together: collecting data in order to respond with efficiency to epidemic. 
At the stage of this research, the simulation's objective is to understand how the DRC's healthcare system reacts in Epidemic diseases. It is a preliminary work before (i) determining metrics to analyze process simulations and (ii) developing modules in embedded systems - phones or tablets- to assist the end-user in the data collection, coupled with the multiagent system. Multiagent-based-simulation (MABS) allows explicitly modeling the behavior of each individual and viewing the emergent system from the interactions between the individuals. Morvan in [37] proposes a survey on MABS and presents several multiagents platforms. In these existing platforms, we have not found solutions which can act as both a simulation system and a tool to end-users on embedded systems.

DIAMOND method and its associated simulator MASH - MultiAgent Software Hardware simulation - developed in LCIS Laboratory provided the capacity of testing a method in a tool of simulation [23, 34].

DIAMOND - Decentralized IterAtive Multiagent Open Network Design - is a method that guides the designer from the requirements to the implementation. It is based on A-E-I-O decomposition for Agent, Environment, Interactions and Organization of the system. The Agent dimension concerns the model of the reactive agent represented by a simple automaton, the cognitive agent manipulating information or a more complex agent based on a knowledge system. The Environment is the context in which the agent reacts, its geographic location. The Interaction dimension specifies the way the agents communicate; it mainly consists of protocols of interaction or the type of communication. The Organization dimension reflects the structural relations between the agents (group, hierarchy, market). These four key concepts are considered under a global angle (the society) and a local one (agent view).

The DIAMOND method proposes an analysis phase of the problem in four steps:

- the situation phase helps to find the society's circumference to be represented by defining the limits of the system, agents and environment;

- the individual phase concerns the internal functioning of the agents (behavior and the knowledge);

- the social phase defines the relations between agents, particularly by integrating into its knowledge communication protocols and information structures to understand the society organization and

- The phase of socialization consists of integrating the individual agents into the society by adding the social influences into its behavior (possible answers in the requests from the outside, the launch of interaction protocols and the choice to be made according to its position in the organization).

We decide to use this method because it allows explicitly designing the behavior of each individual. However, coupled with the MASH simulator platform, it is useful to view the emergent system from the interactions between the individuals. The process in Fig. 3 can be modelized by agents able to be simulated in the MASH simulator. The MASH simulation platform was used to simulate systems with embedded and software agents. It is suitable to our problem because we plan to provide a tool for collecting data with phones or tablets applications. 
To have an individual-centered vision of the process is one of the advantages of this simulation. Afterwards, we will be able to contribute to the improvement of the process with an exterior view by proposing changes and ideas to reduce the response time for example.

This section shows the steps to break down multiagent system's elements.

\subsection{Step 1: Agents' Tasks and Knowledge: The Internal Behavior}

To start the analysis, each individual agent's behavior is studied. It is a way of seeing things at a micro level. The phenomenon at macro level does not change and the process remains the same even if observer's level changes.

The objective is to be able to adjust the behavior of each individual agent and probably to add some skills to certain nodes or node types.

The first step of this approach is to find out what to model as agents from information of the process. In our study context, agents are: health centers, General Referral Hospital, Health areas, Provincial Health Division, any national health entity related to the administrative structures, Health Zone Executive Team and Rapid Ripost Team for human team working group. Figure 4 shows for instance the internal behavior of one agent (Rapid Ripost Team).

For each agent, we have to list all its skills, what information will be required to store or to handle and how agent acquires this information. This information should be acquired directly by perception (e.g. the user grasps something) or on demand by asking other nodes (higher hierarchy or same level nodes).

In this step, we obtain for each agent a vision of the relevant knowledge to perform its individual tasks. That information is required by agents working in the same environment. The result is a set of tasks that an agent can perform. These tasks correspond to the skills of each node. Some skills are executed by one agent without the need of other agents. But to achieve a goal, an agent should have partial information and should ask to other agents to complete their goal. However, this will result to a cooperative behavior in place of an individual one which is entirely internal to agent. This kind of social behavior reflects an interaction between several agents: either to gain information or to share tasks.

\subsection{Step 2: Agent's Sharing Data and Interactions}

Social Behavior. In this second step, we will have to create interactions between nodes for example to back up information (Health Center to Health Zone Executive Team) or to receive orders (Health Zone Executive Team from Provincial Health Division). These interactions should intervene between different partner groups such as health areas. In the implementation, we define very simple interaction protocols for data exchange such as receiving information, answers/queries or order to perform a task. For some tasks, such as health alert surrounding areas, it is no longer just a request for information but cooperative behavior brings into play several kinds of agents. To communicate with others, agent uses interaction protocols. We will there-fore express how this behavior will be realized by defining a more sophisticated interaction protocol than query/response. Various protocols are available for negotiating, giving orders, 


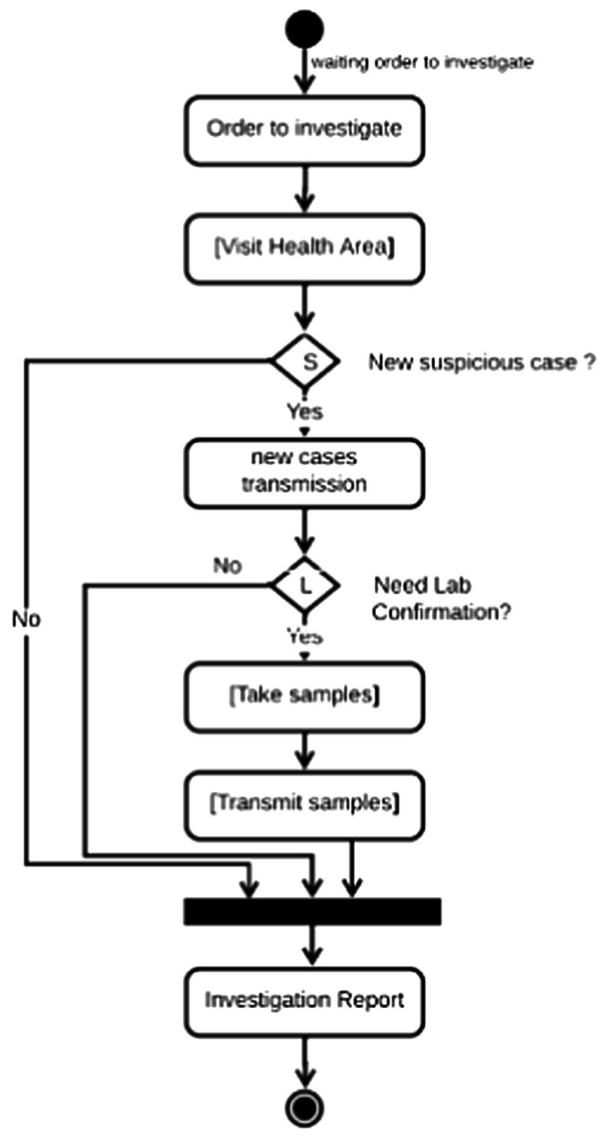

Fig. 4. Investigating an outbreak [1].

waiting for answers. The interaction patterns that will govern this cooperative behavior will be organized between the agents.

Interaction Protocols. The protocol is a part of the agents' knowledge. In our simulation, agents have a list of protocols they should initiate to answer others. For the moment, we use a simple protocol with two states as represented in Fig. 6: "inform" and "inform back". For example, Agent A1 launches an instance of protocol P1, with the state S1. The agent A2 receives a call from A1 with the performative "information" in the state S1. A2 knows the protocol and searches the next transition; it slips into state S2 and sends an acknowledgment to agent A1. A1 treats the message and the conversation finishes. The ACL FIPA compliant Performatives are used:

- RDCMessage.ACL_QUERY_REF for queries/answers,

- RDCMessage.ACL_REQUEST for an order to perform a task and,

- RDCMessage.ACL_INFORM for inform/acknowledgment. 
Position in the Organization. The last step is to consider an agent's position in organization when he initiates interactions with others. An organization should be a hierarchy or a simple group. For example, to alert neighboring Health Zone, health center agents must know the surrounding areas of health zone, which is a group or an organization in the multiagent system, and make a decision based on its position in this group. The agent's position in the organization is integrated in the decision loop.

Agent's Internal Decision Making Loop. The previous steps showed agent's skills, agent's complex behavior (internal and social) and the knowledge of interaction protocols. On the hypothesis that each agent gets this information, we can now build the agent decision loop. On one hand purely individual behavior runs only with an agent's context information and does not need other agents to complete the agent goal. On other hand, social behavior involves relationships between agents. An individualcentered approach defines agent at micro level so that interactions with other agents have to be merged with the internal behavior in the agent's decision loop. The individual and cooperative behaviors are both integrated into the decision loop. In the individual behavior, a set of tasks is launched in the internal decision loop. In its decision loop, the agent should have to respond to the message from others, which are part of interaction protocols initiated by other agents or parts of the agent interaction patterns. These tasks have to be synchronized with the messages received from others agents.

As an external view, huge decision loops, which are decentralized in the several kinds of agents, seem to be synchronized at the system level. But in fact, each agent decides in what state to pass according to its knowledge and the state of its interactions.

\subsection{Step 3: Collaborative Tasks of Agents}

Agents would collaborate to achieve some objectives. To investigate on Health Area, Rapid Ripost Team must wait for an order from Health Zone Executive Team. The later receives health data every week from Health Center and checks if the threshold of the followed pathology has been reached. The same collaboration is needed between Rapid Ripost Team and Health Area, Rapid Ripost Team and Laboratory. The sequence diagram (Fig. 5) gives a snapshot of the kind of collaboration found in agents concerned with an outbreak investigation.

Message Format for Interaction. The messages exchanged between agents contain sender and receiver agents, protocol information and data to manage like [sender; receiver; conversation; perform; protocol; inst_prot; state_prot; data]. The data follow a format according to the performative.

To interact through a message sent by another agent, a simple protocol is established. For instance when Rapid Ripost Team asks a laboratory to perform exams, he has to first check its state to be convinced that it can answer his request. A simple protocol with acknowledgment is used. 


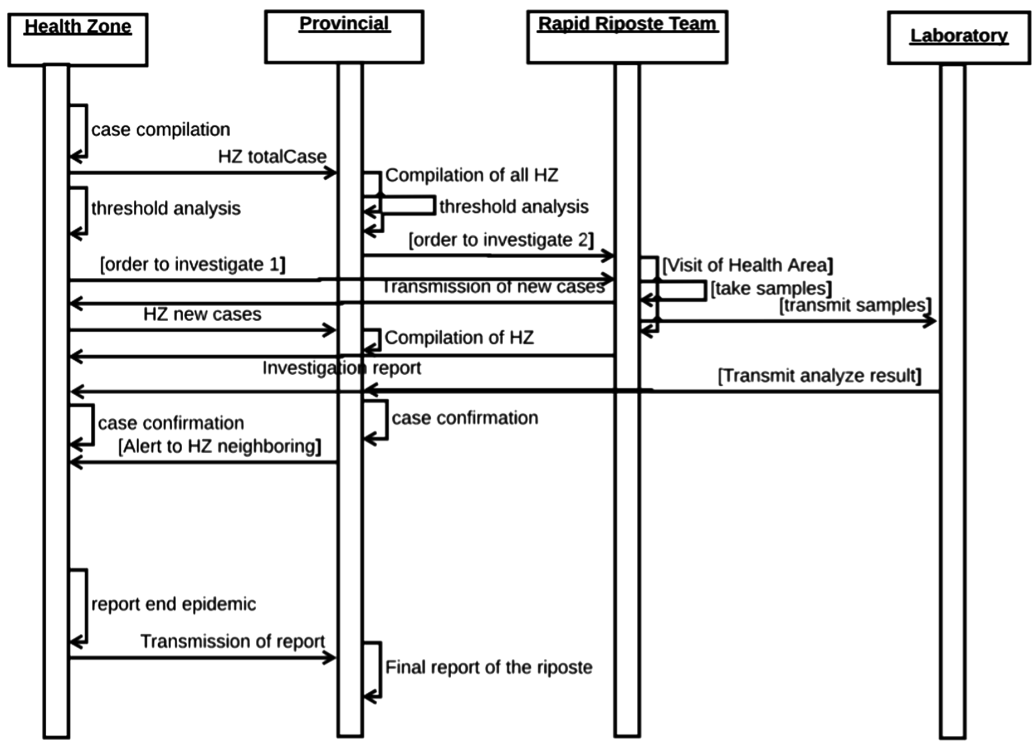

Fig. 5. Sequence diagrams of investigating an outbreak.

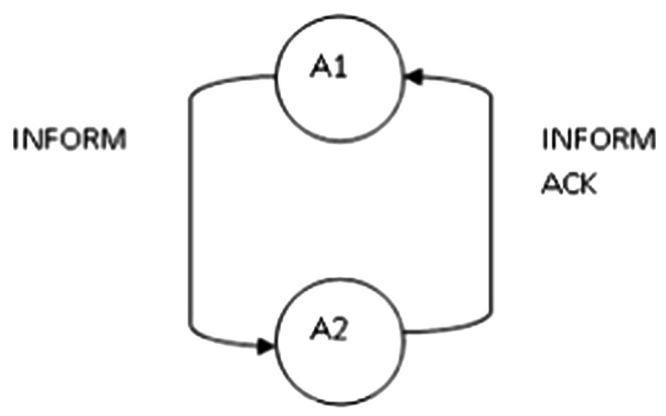

Fig. 6. Protocol for information with acknowledgment [1].

The agent changes state when he asks for information and when he receives answers to his request (Fig. 6). In a future simulation, a negotiation protocol with a call for proposal to several laboratories will be tested. However, the agent launching the conversation should negotiate among laboratories which one is available, near or powerful.

\subsection{Overview of Agents in Process Analysis}

The analyze of the cycle of outbreak response presented on 4.4 isolated some individuals playing different roles to achieve the objectives assigned to the riposte process. A simple class diagram (Fig. 7) gives a quick overview of main actors. 


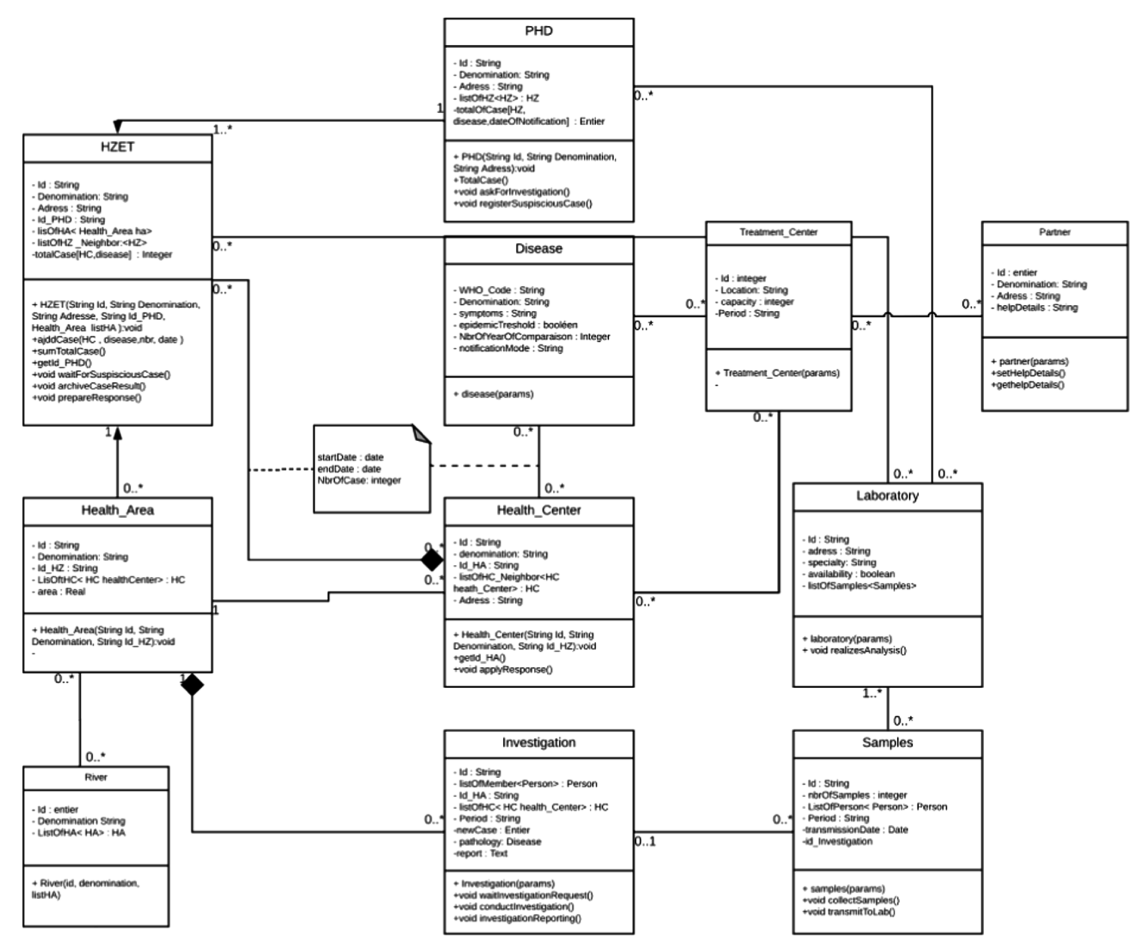

Fig. 7. Main classes resulting from outbreak riposte analyze.

Health Center as agent can exchange message with Agent 'Health Area' to get information about rivers crossing the area of its location. Since this knowledge is crucial to anticipate cholera epidemic in raining season, this collaboration is very important.

Rapid Riposte Team (RRTAgent) investigate on suspicious case in a Health Area which means visiting population and Health Centers found in it If the disease requires laboratory analyzes to confirm the case RRTAgent must take samples and transmit it to LaboratoryAgent available.

\section{Simulation: Cholera Case Study}

\subsection{Cholera: A Plague Still Causing Major Epidemics}

More than one million cases of cholera in Yemen have been reported to the World Health Organization since April 2017. The International Committee of the Red Cross does not hesitate to mention "the worst crisis humanitarian relief in the world "in this country. Despite the International Health Regulations, revised in 2005 to encourage countries to report events constituting a public health emergency, cases remain under- 
reported worldwide due to fear of economic consequences, often insufficient surveillance systems and a lack of standardized terminology to define a case of cholera. ${ }^{2}$

The disease results from the absorption by the mouth of water or food contaminated by the cholera vibrio. After a few hours to a few days of incubation, violent diarrhea and vomiting occur, without fever. In the absence of treatment, death occurs in 1 to 3 days, by cardiovascular collapse (fall in blood pressure) in 25 to $50 \%$ of cases. Mortality is higher among children, the elderly and vulnerable individuals.

The treatment consists essentially of compensating the digestive losses of water and electrolytes. Rehydration is given orally or intravenously, depending on the degree of dehydration. The improvement is noticeable after a few hours and healing, without sequelae, is obtained in a few days. Antibiotic therapy is recommended by WHO only for severe dehydration.

In 1973, since a first outbreak, V. cholerae serogroup O: 1 biotype El Tor was reintroduced several times in the Democratic Republic of Congo. 26 health zones in 6 provinces are identified as sanctuary areas. Kinshasa has recorded several outbreaks of the cholera epidemic. The 2017-2018's episode caused 56 deaths out of 1272 suspicious cases reported, as shown in Fig. 8.

\subsection{Data Collection}

DLM is a disease control direction located in Kinshasa which collects the national data for disease monitoring. It provided us with ten years data of cholera outbreak from 2008 to November 2017. We extracted the data for Kinshasa grouped on its 35 health zones to compare with Provincial Health Division's data.

Provincial Health Division's data contained more details about Health centers that reported the suspicious cases and theirs Health Area. We analyze the data from first January 2017 to December 30th 2017.

\subsection{Methods}

We reported cumulated data of each health zone to a map, as shown in Fig. 8, to find their neighboring and try to suggest the best way to establish collaboration between them in order to stop disease propagation.

We focus on two groups of health zones. In the first group we have Binza-Météo, Mont-Ngafula, Kokolo and Kintambo. The second contains Limete and Kingabwa.

Our hypothesis was: if actors from each heath zone could exchange disease information with their neighbors as soon as an outbreak happens, it would be possible to reduce the propagation. For example, epidemic began at Kintambo on January 2017. As the communication and sensitization weren't establish with its neighbors, some weeks after disease was reported from Kokolo and Binza-Météo.

\footnotetext{
$\overline{2}$ https://www.pasteur.fr/fr/journal-recherche/dossiers/cholera-fleau-encore-origine-epidemiesmajeures.
} 


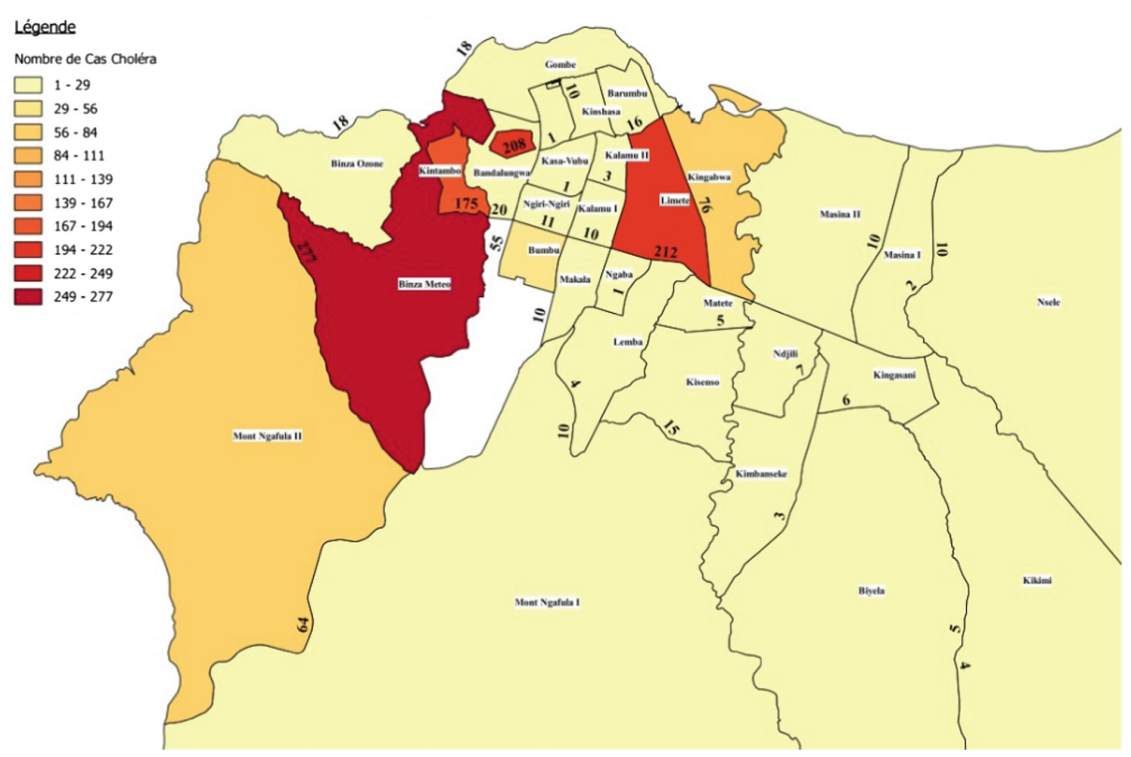

Fig. 8. From 01/01/2017 to 30/04/2018, Kinshasa's Health zones reported 1272 cases of Cholera outbreak with 56 dead.

\subsection{Results}

To test and evaluate our approach, we adapted MASH simulator developed by LCIS Lab for a wireless sensor multiagent system [34]. We focus our simulation to these health zones: Binza-Météo, Mont-Ngafula, Kokolo, Kintambo, Limete and Kingabwa.

The simulation concerns precisely Kintambo and Limete that register more suspicious case of cholera outbreak during 2017 and from them other neighboring health zones were affected. The main idea is to see how the future system would react if each actor of health system could perform its own task with autonomy. These experiences could result to many scenarios and the best of them will be proposed to DRC's Health System to reduce decision time as each actor can execute his talks according to the knowledge of the environment and the outbreak determinants he will be provided with.

We chose two Health-Zones of Kinshasa Provincial Health Division for the simulation. Kintambo and Limete are Health Zones that register respectively 57 and 64 suspicious cases with death in 2017. The epidemic began from them and the propagation of disease affected their neighbors with an important amount of suspicious cases. Kokolo counted 208 cases while Binza-météo registered 153 suspicious cases at the same period.

To respond to an outbreak noticed at a Health Center, health staff of the concerning Health Center must refer to Health Zone Executive Team. In their turn, Health Zone Executive Team must refer to Provincial Health Division and Provincial Health Division to central level. This chain of hierarchical contacts can enlarge time decision.

In our simulation, we worked with these hypotheses: (i) each Health Center actor must contact immediately its Health Executive Team as soon as it encounted a 
suspicious case; (ii) Health Zone Executive Team cumulate suspicious case and create an RRT when the disease's threshold is reached; (iii) RRT can contact the nearest LaboAgent able to answer to his request or to use his information to make decision. We considered twenty-eight Health Zone Executive Team in yellow or red, forty seven Health Center in green or blue, RRT in grey one Provincial Health Division and one medical test laboratories (LaboAgent). The first suspicious case was detected in Health Center \#49 in green. Figure 9 illustrates those actors working as Agents. The below map represents Health zones in north of Kinshasa.

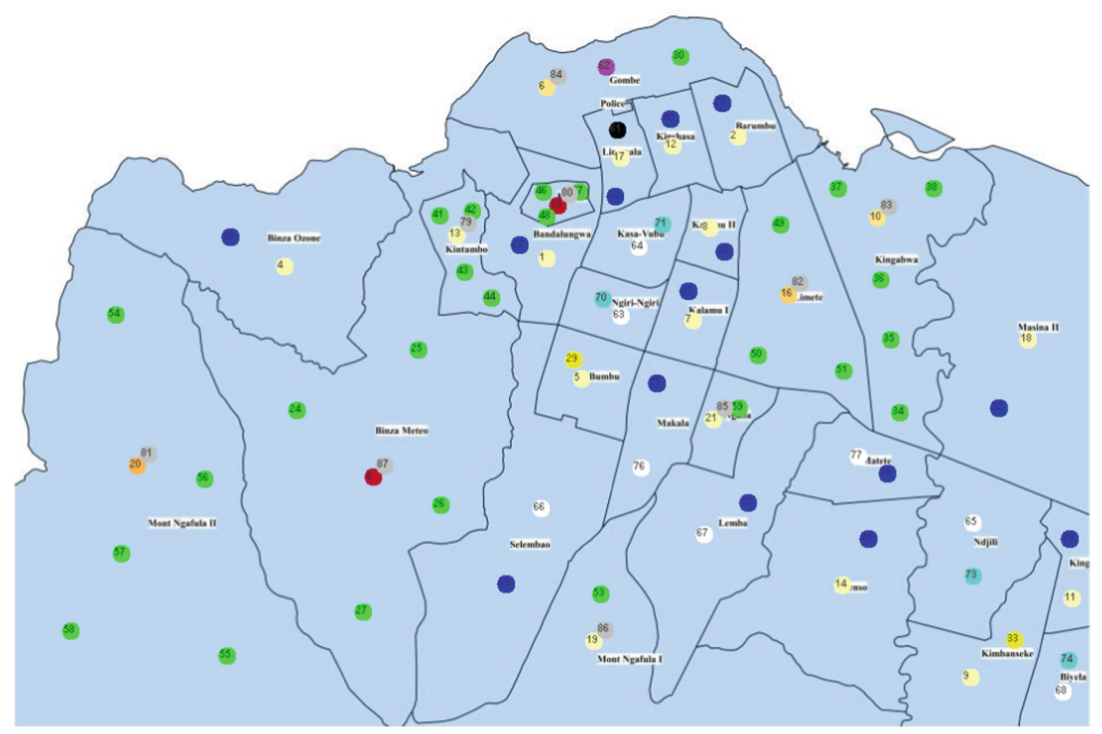

Fig. 9. Suspicious cases of cholera outbreak reported during 2017 by Kinshasa's Health Zones. (Color figure online)

The simulation trace file (Fig. 10) shows the communication between agents. As they are autonomous they perform their own tasks like "cumulate new cases", "conduct the investigation on Health Zone \# 16" and manage messages like "realize analyzes from Health Center", "receive sample to_ analyze" or "report suspicious case detected". HZET Agents (\#16 and \#13) are waiting for a suspicious case message from any HC. Whenever Health Zone Executive Team Agent receives such a message he computes cases and compare with the threshold of disease monitoring to decide the necessity of building a RRT Agent able to investigate in Health Centers around suspicious case in Health Area. The visited health center will turn from green to blue color. RRT Agent could send samples to LaboAgent while searching for new cases in Health Areas. The organization of outbreak riposte could depend on the results from LaboAgent and investigation report from RRT Agent. 
The agents operate independently: HCAgent transmits data to Health Zone Executive TeamAgent, RRTAgent completes a full investigation, LaboAgent conducts medical testing and transmits results to HZETAgent and PHDAgent which manages all information from Health Zone Executive Team under its supervision. Message synchronization between kinds of agent is done in the agent's decision loop. A protocol with two states is used and implements KQML-like performatives. The four numbers in the message are " 1 " for inform (give information), " 2 " for query information (ask for an information) and " 3 " for request (ask for a task to be done). The agents communicate and achieve their goal by reacting to messages from others or executing their inner task as response to a query.

With positive results from LaboAgent, Health Zone Executive Team sends warning and preventive measures to his all Health Centers. PHDAgent can also alert the surrounding Health Zone Executive Teams.

\section{Conclusions}

In this paper, we have showed how multi-agent system can improve the organizations' tasks in order to decrease the time to response when an epidemic disease is suspected or detected. A main research result can be highlighted: the use of a multi-agent method previously dedicated to wireless sensors and applied to human organizations.

We have proved that the method was generic enough and gave good results with real data and a hierarchical and complex health eco-system. MAS is often used in health domain and our paper's result complete the panel of applications with real data and under eco-system constraints.

Some limits must be underlined: (i) in order to adapt the method, we have defined hypothesis that strongly constraint the models; (ii) the stakeholders have been introduced in the method only with their job characteristics and adding their experience in the simulation can probably enhance the results. Nevertheless the multiagent method can cover only one part of the global problem. We discussed about an organizational method useful to enlarge all aspects of the problem laid down.

We propose in the future works to widen the approach to take into account different and complementary aspects: health data collection and transmission, health data quality, improvement of the complete riposte process, improvement of the health system organization. In order to, we propose a three-phases innovation method named Chicken useful to supervise and improve the epidemic disease riposte:

- Phase 1: define the life cycle of the epidemic disease to watch over.

- Phase 2: health data monitoring

- Phase 3: riposte and feed-back

Each phase of this innovation method is build with models, methods' fragments, tools coming from different sciences domains and proposes a road map to improve the riposte and the health data quality. Then the multiagent method becomes a method's fragment in the wider method Chicken. 


\section{References}

1. Tshilenge Mfumu, J.-C., Mercier, A., Verdier, C., Occello, M.: Towards an agent-based model to monitor epidemics and chronic diseases in DR congo, pp. 83-93 (2018)

2. Programme des Nations Unies pour le Développement: Rapport sur le développement humain (2016). http://hdr.undp.org/sites/default/files/HDR2016_FR_Overview_Web.pdf. Accessed 05 Apr 2018

3. World health statistics 2014: Geneva: World Health Organization (2014)

4. Recueil des normes de la zone de santé: Ministère de la Santé (2006)

5. Weltgesundheitsorganisation (ed.): Règlement sanitaire international: 2005, 2nd edn. Organisation Mondiale de la Santé, Genève (2006)

6. Ministère de la Santé, RDC: Guide Technique pour la surveillance intégrée de la maladie et riposte (2011). http://www.luttecontrelamaladie.org/bvf/GUIDE\%20SMIR.pdf. Accessed 26 Feb 2018

7. Greenberg, A.: ICTs for Poverty Alleviation: Basic Tool and Enabling Sector (2005)

8. Wouters, B., Barjis, J., Maponya, G., Martiz, J., Mashiri, M.: Supporting home based health care in South African rural communities using USSD technology. In: AMCIS 2009 Proceedings, p. 410 (2009)

9. Zurovac, D., Talisuna, A.O., Snow, R.W.: Mobile phone text messaging: tool for malaria control in Africa. PLoS Med. 9(2), e1001176 (2012)

10. Mutale, W., et al.: improving health information systems for decision making across five sub-Saharan African countries: implementation strategies from the African Health Initiative. BMC Health Serv. Res. 13(2), S9 (2013)

11. Aker, J.C., Mbiti, I.M.: Mobile phones and economic development in Africa. J. Econ. Perspect. 24(3), 207-232 (2010)

12. Lester, R.T., et al.: Effects of a mobile phone short message service on antiretroviral treatment adherence in Kenya (WelTel Kenya1): a randomised trial. Lancet Lond. Engl. 376 (9755), 1838-1845 (2010)

13. Zurovac, D., et al.: The effect of mobile phone text-message reminders on Kenyan health workers' adherence to malaria treatment guidelines: a cluster randomised trial. Lancet Lond. Engl. 378(9793), 795-803 (2011)

14. Wooldridge, M., Jennings, N.R., Kinny, D.: A methodology for agent-oriented analysis and design. In: Proceedings of the Third Annual Conference on Autonomous Agents, New York, NY, USA, pp. 69-76 (1999)

15. Kinny, D., Georgeff, M., Rao, A.: A methodology and modelling technique for systems of BDI agents. In: Van de Velde, W., Perram, J.W. (eds.) MAAMAW 1996. LNCS, vol. 1038, pp. 56-71. Springer, Heidelberg (1996). https://doi.org/10.1007/BFb0031846

16. Brazier, F.M.T., Dunin-Keplicz, B.M., Jennings, N.R., Treur, J.: Desire: modelling multiagent systems in a compositional formal framework. Int. J. Coop. Inf. Syst. 06(01), 67-94 (1997)

17. Demazeau, Y.: "Voyelles," Mém. D’habilitation À Dir. Rech. INP Grenoble (2001)

18. Wooldridge, M.: The Gaia Methodology for Agent-Oriented Analysis and Design, p. 28

19. DeLoach, S.A.: Analysis and Design using MaSE and agentTool. Air Force Inst of Tech Wright-Patterson AFB, OH, School of Engineering and Management, April 2001

20. Padgham, L., Winikoff, M.: Prometheus: a methodology for developing intelligent agents. In: Giunchiglia, F., Odell, J., Weiß, G. (eds.) AOSE 2002. LNCS, vol. 2585, pp. 174-185. Springer, Heidelberg (2003). https://doi.org/10.1007/3-540-36540-0_14

21. Rao, A.S., Georgeff, M.P.: BDI Agents: From Theory to Practice, p. 8 (1995) 
22. Mea, V.D.: Agents acting and moving in healthcare scenario - a paradigm for telemedical collaboration. IEEE Trans. Inf Technol. Biomed. 5(1), 10-13 (2001)

23. Jamont, J.-P., Occello, M.: Designing embedded collective systems: The DIAMOND multiagent method. In: IEEE International Conference on Tools with Artificial Intelligence ICTAI 2007, Patras, Greece, pp. 91-94 (2007)

24. Weber, A.: Modélisation et Gestion de Flux par Systèmes Multiagents: Application à un système d'aide à la décision en épidémiologie, Ecole Centrale de Lille (2007)

25. Mercier, A., Raievsky, C., Occello, M., Genthial, D.: Solutions multi-agents pour la prise en charge à domicile des séniors. Ingénierie Systèmes Inf. 18(6), 83-112 (2013)

26. Younsi, F.-Z:: Mise en place d'un Système d'Information Décisionnel pour le suivi et la prévention des épidémies. Lyon (2016)

27. Bae, J.W., et al.: Evaluation of disaster response system using agent-based model with geospatial and medical details. IEEE Trans. Syst. Man Cybern. Syst. 48, 1-16 (2017)

28. Han, B.-M., Song, S.-J., Lee, K.M., Jang, K.-S., Shin, D.-R.: Multi-agent system based efficient healthcare service. In: 2006 8th International Conference Advanced Communication Technology, vol. 1, pp. 5-51 (2006)

29. Bergenti, F., Poggi, A., Tomaiuolo, M.: Using multi-agent systems to support e-health services. In: Handbook of Research on ICTs for Human-Centered Healthcare and Social Care Services, pp. 549-567 (2013)

30. Jemal, H., Kechaou, Z., Ayed, M.B., Alimi, A.M.: A multi agent system for hospital organization. Int. J. Mach. Learn. Comput. 5(1), 51-56 (2015)

31. Bergenti, F., Poggi, A., Tomaiuolo, M.: Multi-agent systems for e-health and telemedicine. In: Encyclopedia of e-Health and Telemedicine, pp. 688-699 (2016)

32. Koutkias, V.G., Chouvarda, I., Maglaveras, N.: A multiagent system enhancing home-care health services for chronic disease management. IEEE Trans. Inf Technol. Biomed. 9(4), 528-537 (2005)

33. Shakshuki, E., Reid, M.: Multi-agent system applications in healthcare: current technology and future roadmap. Proc. Comput. Sci. 52, 252-261 (2015)

34. Jamont, J.-P., Occello, M.: A multiagent tool to simulate hybrid real/virtual embedded agent societies. In: Proceedings of the 2009 IEEE/WIC/ACM International Joint Conference on Web Intelligence and Intelligent Agent Technology, Washington, DC, USA, vol. 02, pp. 501-504 (2009)

35. Smaradottir, B., Martinez, S., Holen-Rabbersvik, E., Vatnøy, T., Fensli, R.: Usability Evaluation of a Collaborative Health Information System - Lessons from a User-centred Design Process (2016)

36. Ministère du Plan de la RD Congo: Deuxième Enquête Démographique et de santé 20132014, September 2014. https://www.unicef.org/drcongo/french/00_-_00_-_DRC_DHS_ 2013-2014_FINAL_PDF_09-29-2014.pdf. Accessed 27 Feb 2018

37. Morvan, G.: Multi-level agent-based modeling - a literature survey. ArXiv12050561 Cs, May 2012 